\title{
Diffraction by random Ronchi gratings
}

\author{
Francisco Jose Torcal-Milla* and Luis Miguel SancheZ-Brea \\ 1Applied Optics Complutense Group, Optics Department, Facultad de Ciencias Físicas, Universidad Complutense de Madrid, Plaza de las Ciencias \\ 1, 28040, Madrid (Spain) \\ *Corresponding author: fjtorcal@ucm.es
}

Received XX Month XXXX; revised XX Month, XXXX; accepted XX Month XXXX; posted XX Month XXXX (Doc. ID XXXXX); published XX Month XXXX

In this work we obtain analytical expressions for the near and far field diffraction of random Ronchi diffraction gratings where the slits of the grating are randomly displaced around their periodical positions. We theoretically show that the effect of randomness in the position of the slits of the grating produces smoothing and disappearance of the self-images at the near field. On the other hand, it cancels high order harmonics in far field, resulting in only a few central diffraction orders. Numerical simulations by means of the Rayleigh-Sommerfeld diffraction formula are performed in order to corroborate the analytical results. These results are of high interest for industrial and technological applications where manufacture errors need to be considered. (C) 2016 Optical Society of America

OCIS codes: (050.0050) Diffraction and gratings; (050.1950) Diffraction gratings; (030.6600) Statistical Optics.

http://dx.doi.org/10.1364/AO.99.099999

\section{INTRODUCTION}

Diffraction gratings are optical elements commonly used in many different branches of science and applications, 1 . We can find diffraction gratings in many diverse fields as optical metrology, laser array illumination, moiré interferometry, spectroscopy, phase locking of laser arrays, etc, 2-5. The most common types of diffraction gratings are amplitude-based and phase-based gratings that modulate the amplitude and the phase of the incident beam respectively, 1 , but other kinds of gratings such as polarization gratings or rough gratings have been introduced in recentyears. Rough gratings can be understood as a modulation of the coherence state of the beam, 6-8. Concerning fabrication methods, gratings are commonly manufactured by direct laser writing, laser ablation or photolithographic methods. The most adequate method for fabrication depends on the substrate of the grating, the desired grating type, the needed accuracy, the desired feature, and so on. For example, gratings over steel substrates are commonly manufactured by laser ablation, 9, and gratings over glass substrates are usually fabricated by photolithographic methods, 10 . Despite manufacturing processes have been improved year after year, manufacturing errors are still present and become more representative when period of the gratings are smaller. The effect of different kinds of imperfections in diffraction gratings such as imperfections in the edge of the slits 11 , random distribution of phase delays 12 , roughness on the surface, 13-15, missing slits, 16 , random distributions 17 , etc, have been analyzed in recent years. In this manuscript, we analytically and numerically investigate the near field diffraction of random Ronchi gratings formed by an ensemble of transparent slits that are not equally spaced but their positions present a certain randomness around their nominal periodical positions. As it has been introduced before, diffraction gratings behavior has been studied from many points of view. Particularly, it is well known that Talbot effect is produced at the near field, consisting of the replication of the grating pattern at different distances from the grating, called Talbot distances, 18-20. The Talbot distance is defined as $z T=2 p^{2} / \lambda$ where $p$ is the period of the grating and $\lambda$ is the illumination wavelength. In our case, as we demonstrate following, the near field intensity produced by the proposed grating is slightly different. Randomness affects to the smoothness of the selfimages of the grating. Besides, the contrast of the self-images decreases in terms of the randomness level, producing the disappearance of the self-images for high randomness. In addition, numerical simulations made by means of a numerical integration of the Rayleigh-Sommerfeld formula corroborate the analytical results. Finally, far field analysis reveals the same behavior as near field, cancelling high diffraction orders for high randomness levels.

\section{NEAR FIELD APPROACH}

Let us consider a one-dimensional diffraction grating whose transmittance is defined as the summation of transparent slits

$$
t\left(x^{\prime}\right)=\sum_{n=-\infty}^{\infty} \operatorname{rect}\left(\frac{x^{\prime}-n p}{\tau}\right)= \begin{cases}1 & n p-\tau / 2 \leq x^{\prime} \leq n p+\tau / 2 \\ 0 & \text { others }\end{cases}
$$

where $n$ are integer numbers, $p$ is the period of the grating and $\tau$ is the opening width of the grating. Now, we include a certain randomness into the central position of each aperture but maintaining its size as a constant. Mathematically, it can be expressed as

$t_{r}\left(x^{\prime}\right)=\sum_{n,-\infty}^{\infty} \operatorname{rect}\left(\frac{x^{\prime}-n p+\alpha_{n}}{\tau}\right)= \begin{cases}1 & n p-\tau / 2+\alpha_{n} \leq x^{\prime} \leq n p+\tau / 2+\alpha_{n} \\ 0 & \text { others }\end{cases}$

(2) 
where $\alpha_{n}$ is a random number following a certain probabilistic distribution. We show in Fig. 1 an example of the grating proposed (dashed line) and a periodical grating of the same period (solid line).

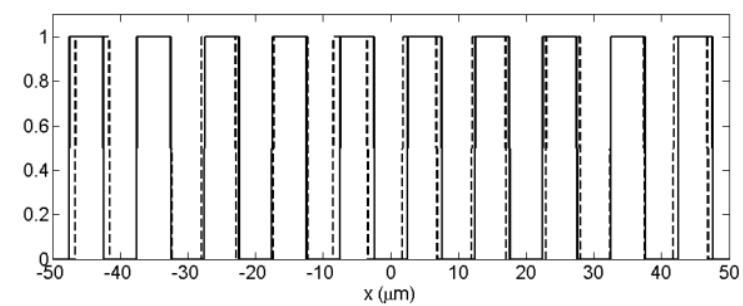

Fig. 1. Example of diffraction grating proposed (dashed line) versus periodical grating (solid line), $p=10 \mu m, w=p / 10$.

The field at a distance $z$ close to the grating can be easily calculated by using the Fresnel approach

$$
\begin{aligned}
U(x, z) & =A \frac{\exp (i k z)}{\sqrt{i k \lambda}} \int_{-\infty}^{\infty} t_{r}\left(x^{\prime}\right) \exp \left[\frac{i \pi}{\lambda z}\left(x-x^{\prime}\right)^{2}\right] d x^{\prime} \\
& =A \frac{\exp (i k z)}{\sqrt{i k \lambda}} \int_{-\infty}^{\infty} \sum_{n=-\infty}^{\infty} \operatorname{rect}\left(\frac{x^{\prime}-n p+\alpha_{n}}{\tau}\right) \\
& \times \exp \left[\frac{i \pi}{\lambda z}\left(x-x^{\prime}\right)^{2}\right] d x^{\prime},
\end{aligned}
$$

where $\lambda$ is the wavelength of the incident light, $A$ is the amplitude of the field and $x$ is the transversal coordinate at the observation plane. Applying Eq. (2) to Eq. (3), it may be rewritten as

$$
U(x, z)=A \sum_{n=-\infty}^{\infty} \int_{n p-\tau / 2+\alpha_{n}}^{n p+\tau / 2+\alpha_{n}} \exp \left[\frac{i \pi}{\lambda z}\left(x-x^{\prime}\right)^{2}\right] d x^{\prime} .
$$

Solving Eq. (4), the field at a distance $z$ in terms of $\alpha_{n}$, results in

$$
\begin{aligned}
U\left(x, z, \alpha_{n}\right)= & \frac{A}{2} \exp (i k z) \sum_{n=-\infty}^{\infty}\left\{\operatorname{erf}\left[\left(-\frac{1-i}{\sqrt{2}}\right) \sqrt{\frac{\pi}{\lambda z}}\left(x-\frac{\tau}{2}-n p-\alpha_{n}\right)\right]\right. \\
& \left.-\operatorname{erf}\left[\left(-\frac{1-i}{\sqrt{2}}\right) \sqrt{\frac{\pi}{\lambda z}}\left(x+\frac{\tau}{2}-n p-\alpha_{n}\right)\right]\right\},
\end{aligned}
$$

where $\operatorname{erf}(\chi)$ is the error function defined as $\operatorname{erf}(\chi)=\frac{2}{\sqrt{\pi}} \int_{0}^{\chi} e^{-t^{2}} d t$.

As we have mentioned before, $\alpha_{n}$ are statistical variables. Therefore, we need to apply an averaging process to calculate the intensity distribution at the near field. The average intensity can be calculated by solving

$$
\begin{aligned}
\langle I(x, z)\rangle & =\left\langle U\left(x, z, \alpha_{n}\right) U^{*}\left(x, z, \alpha_{m}\right)\right\rangle \\
& =\int_{-\infty}^{\infty} \int_{-\infty}^{\infty} p\left(\alpha_{n}, \alpha_{m}\right) U\left(x, z, \alpha_{n}\right) U^{*}\left(x, z, \alpha_{m}\right) d \alpha_{n} d \alpha_{m},
\end{aligned}
$$

where $p\left(\alpha_{n}, \alpha_{m}\right)$ is the joint probability density function of both distributions, that we have chosen Gaussian, 21. In our case, considering that all random variables, $\alpha_{n}$, are uncorrelated, then $p\left(\alpha_{n}, \alpha_{m}\right)=p\left(\alpha_{n}\right) p\left(\alpha_{m}\right)$. Thus, the average intensity can be expressed as

$$
\begin{aligned}
\langle I(x, z)\rangle= & \left\langle U\left(x, z, \alpha_{n}\right) U^{*}\left(x, z, \alpha_{m}\right)\right\rangle \\
& =\int_{-\infty}^{\infty} p\left(\alpha_{n}\right) U\left(x, z, \alpha_{n}\right) d \alpha_{n} \int_{-\infty}^{\infty} p\left(\alpha_{m}\right) U^{*}\left(x, z, \alpha_{m}\right) d \alpha_{m},
\end{aligned}
$$

where

$$
p\left(\alpha_{j}\right)=\frac{1}{\sqrt{2 \pi} w} \exp \left(\frac{-\alpha_{j}^{2}}{2 w^{2}}\right) ; j=n, m,
$$

where $w$ is the standard deviation of the random displacement of the slits around their nominal positions, 21 .

Placing all terms into Eq. (7) it results into

$$
\begin{aligned}
\langle I(x, z)\rangle & =\frac{A^{2}}{8 \pi w^{2}} \sum_{n, m} \int_{-\infty}^{\infty} \exp \left(\frac{-\alpha_{n}^{2}}{2 w^{2}}\right)\left[\operatorname{erf}\left(\varepsilon_{n}+\mu \alpha_{n}\right)-\operatorname{erf}\left(\theta_{n}+\mu \alpha_{n}\right)\right] d \alpha_{n} \\
& \times \int_{-\infty}^{\infty} \exp \left(\frac{-\alpha_{m}^{2}}{2 w^{2}}\right)\left[\operatorname{erf}\left(\varepsilon_{m}^{*}+\mu^{*} \alpha_{m}\right)-\operatorname{erf}\left(\theta_{m}^{*}+\mu^{*} \alpha_{m}\right)\right] d \alpha_{m},
\end{aligned}
$$

where $\quad \varepsilon_{n}=\left(\frac{1-i}{\sqrt{2}}\right) \sqrt{\frac{\pi}{\lambda z}}\left(\frac{\tau}{2}+n p-x\right), \quad \theta_{n}=\left(-\frac{1-i}{\sqrt{2}}\right) \sqrt{\frac{\pi}{\lambda z}}\left(\frac{\tau}{2}-n p+x\right)$, $\mu=\left(\frac{1-i}{\sqrt{2}}\right) \sqrt{\frac{\pi}{\lambda z}}$, and ()$^{\star}$ denotes complex conjugated.

After performing the integrals and simplifying, the mean intensity results

$$
\begin{aligned}
\langle I(x, z)\rangle= & \frac{A^{2}}{4} \sum_{n, m}\left\{\operatorname{erf}\left[\frac{\varepsilon_{n}}{\sqrt{1+2(w \mu)^{2}}}\right]-\operatorname{erf}\left[\frac{\theta_{n}}{\sqrt{1+2(w \mu)^{2}}}\right]\right\} \\
& \left.\times \operatorname{erf}\left[\frac{\varepsilon_{m}^{*}}{\sqrt{1+2\left(w \mu^{*}\right)^{2}}}\right]-\operatorname{erf}\left[\frac{\theta_{m}^{*}}{\sqrt{1+2\left(w \mu^{*}\right)^{2}}}\right]\right\} .
\end{aligned}
$$

We introduce again all variables for a better understanding of the result,

$$
\begin{aligned}
\langle I(x, z)\rangle & =\mid \frac{A}{2} \sum_{n}\left\{\operatorname{erf}\left[\sqrt{\frac{\pi}{2 \pi w^{2}+i z \lambda}}\left(n p-x+\frac{\tau}{2}\right)\right]\right. \\
& -\operatorname{erf}\left[\sqrt{\left.\left.\frac{\pi}{2 \pi w^{2}+i z \lambda}\left(n p-x-\frac{\tau}{2}\right)\right]\right)\left.\right|^{2}}\right.
\end{aligned}
$$

As can be observed, it almost corresponds to the diffraction produced by an ensemble of slits of size $\tau$ regularly placed at distances multiple of $p$ but with an extra term due to randomness, $2 \pi w^{2}$. For $w \rightarrow 0$ we recover the classical diffraction produced by an ensemble of slits regularly placed.

We show in Fig. 2 some examples of the near field intensity produced by the grating proposed with the following parameters, $p=20 \mu \mathrm{m}$, 
$\tau=p / 2$, 51 slits, $\lambda=630 \mathrm{~nm}, A=1$, and $w=0$ (Fig. 2a), $w=p / 10$ (Fig. 2b), $w=p / 5$ (Fig. 2c), and $w=p / 2$ (Fig. 2d).
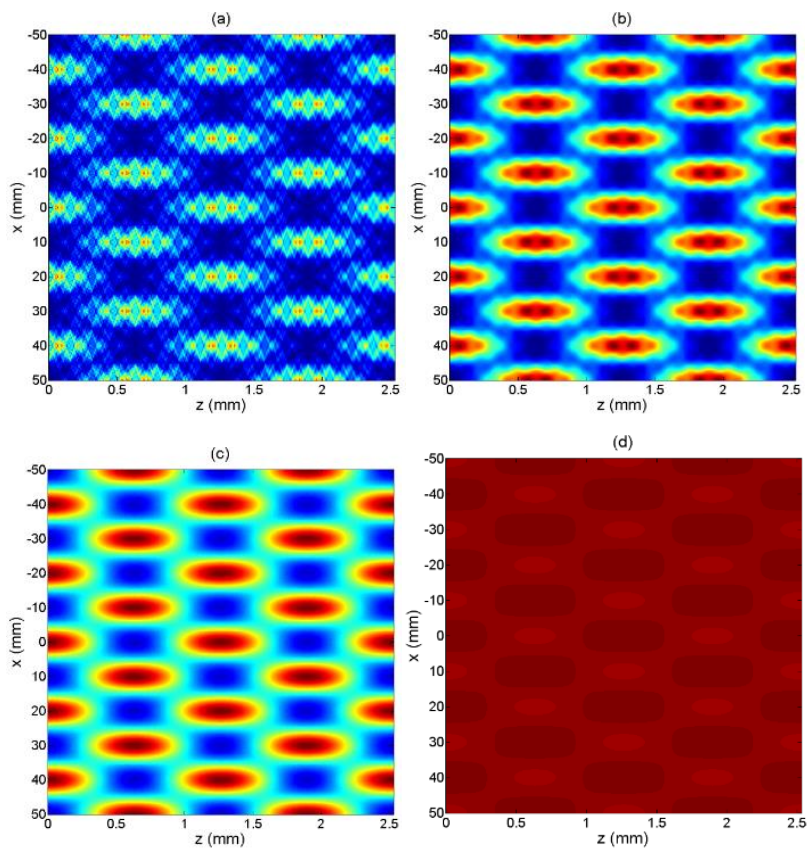

Fig. 2. Analytical self-images calculated by using Eq. (11) with a grating of nominal period $p=20 \mu \mathrm{m}$ illuminated by a plane wave of wavelength $\lambda=630 \mathrm{~nm}$. The amounts of randomness are: (a) $w=0$, (b) $w=p / 10$, (c) $w=p / 5$, and (d) $w=p / 2$.

As can be observed, randomness of the grating produces smoothing of the self-images. For a better observation, we show in Fig. 3 (asterisks) the first self-image and the corresponding fitting to a sinusoidal (solid line) corresponding to Fig. 2. The Root Mean Squares of these fittings are $R M S E=0.2181(w=0)$, $R M S E=0.0882(w=p / 10)$,

$R M S E=0.02956(w=p / 5)$, and $R M S E=7.448 \times 10^{-6}(w=p / 2)$, which reveals better fitting for higher randomness. The effect of the randomness on the grating structure produces smoothing of the selfimages in terms of $w$. Indeed, for values over $w \approx p / 2$, the self-images disappear completely. On the other hand, for the limit case $w \rightarrow 0$, we recover the intensity produced by a periodical diffraction grating at the near field.

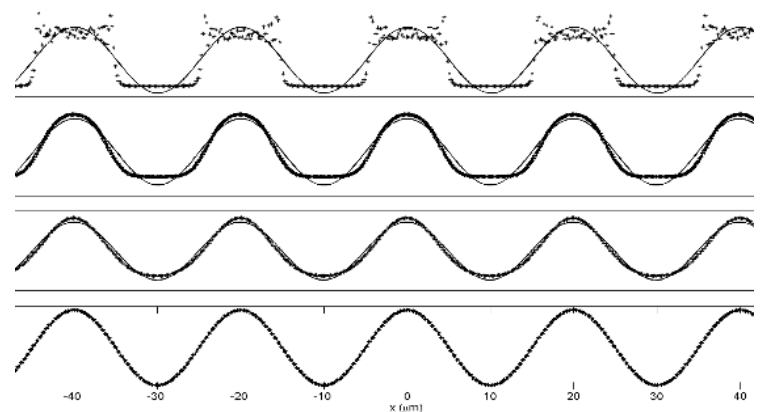

Fig. 3. First self-image corresponding to Fig. 2. Asterisk represent analytical results and solid lines represent fittings to sinusoidal.

In addition, we show in Fig. 4 the contrast of the self-images in terms of $w$ (solid line) following the definition of contrast given in 22,

$$
C(w)=\frac{\left\langle I_{\max }\right\rangle-\left\langle I_{\min }\right\rangle}{\left\langle I_{\max }\right\rangle+\left\langle I_{\min }\right\rangle},
$$

where

$$
\left\langle I_{\max }\right\rangle=\int g(x)\left\langle I\left(x, z_{T}\right)\right\rangle d x,
$$

$\left\langle I_{\min }\right\rangle=\int[1-g(x)]\left\langle I\left(x, z_{T}\right)\right\rangle d x$, and $g(x)$ is the grating profile without randomness. Besides, we show in Fig. 4 (dot line) the contrast calculated by using the classical contrast definition, $\hat{C}(w)=\left(\hat{I}_{\max }-\hat{I}_{\min }\right) /\left(\hat{I}_{\max }+\hat{I}_{\min }\right)$, where $\hat{I}_{\max }$ is the maximum intensity and $\hat{I}_{\text {min }}$ is the minimum intensity. It decreases following a gaussian function and reaches zero for $w \approx 0.5 p$. We have tested that this value is independent on the period of the grating and the wavelength.

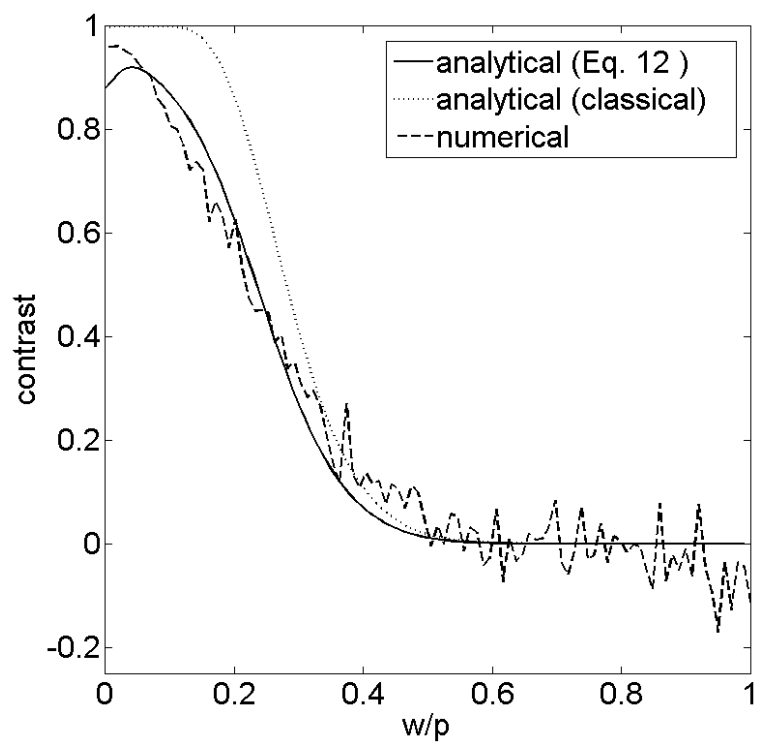

Fig. 4. Contrast of the first self-image in terms of the randomness. $p=20 \mu \mathrm{m}$. Analytical calculated by using Eq. (12) (solid line), analytical using the classical definition of contrast (dot line), and numerical by using the Rayleigh-Sommerfeld approach (dashed line).

\section{FAR FIELD APPROACH}

In this case, let us consider the Fraunhofer diffraction formula. The field produced by the random grating is calculated in a similar way as for near field propagation just solving the following integral expression

$U_{F}(x, z)=A \sum_{n=-\infty}^{\infty} \int_{n p-\tau / 2+\alpha_{n}}^{n p+\tau / 2+\alpha_{n}} \exp \left[\frac{2 i \pi}{\lambda z} x x^{\prime}\right] d x^{\prime}$.

Thus, the field results in

$$
U_{F}(x, z)=A \tau \sum_{n=-\infty}^{\infty} \operatorname{sinc}\left(\frac{\pi \tau}{\lambda} \theta\right) \exp \left(-\frac{2 i \pi p}{\lambda} n \theta\right) \exp \left(-\frac{2 i \pi}{\lambda} \alpha_{n} \theta\right) \text {, }
$$

where $\operatorname{sinc}(\xi)=\sin (\xi) / \xi$ and $\theta=x / z$. We again use the density probability functions, Eq. (8), to obtain the mean intensity

$$
\left\langle I_{F}(x, z)\right\rangle=(A \tau)^{2} \sum_{n=-\infty}^{\infty} \operatorname{sinc}^{2}\left(k \frac{\tau}{2} \theta\right) \exp [i p k(m-n) \theta] \exp (k w \theta)^{2}
$$

(15) 


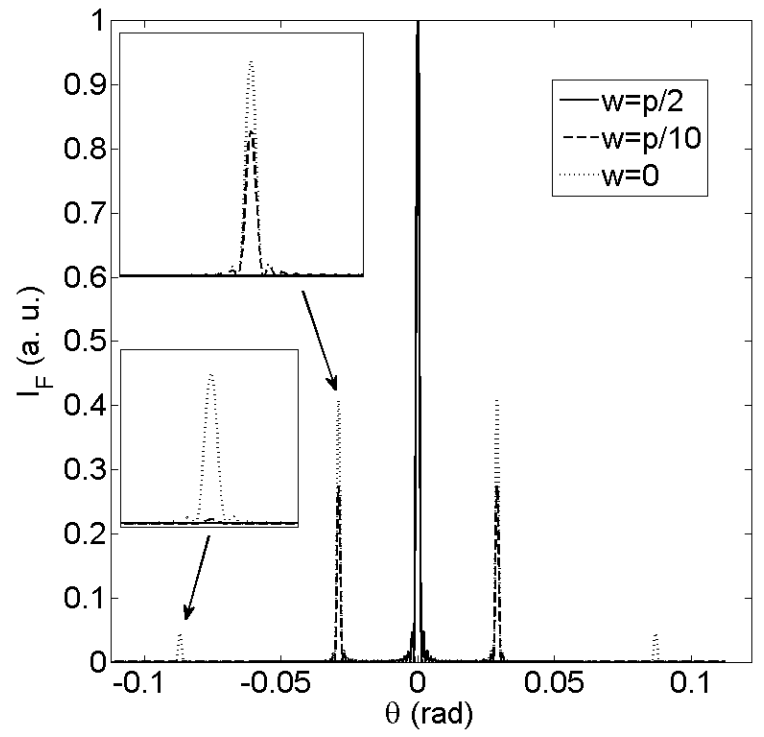

Fig. 5. Far field mean intensity distribution corresponding to a random diffraction grating of period $p=20 \mu m$ illuminated by a plane wave of wavelength $\lambda=630 \mathrm{~nm}, w=0$ (dot line), $w=p / 10$ (dashed line), and $w=p / 2$ (solid line).

It corresponds to the classical solution but adding an extra term depending on the amount of randomness, $w$, that modulates the intensity. For relatively high $w$, high harmonics disappears, remaining only zero and firsts diffraction orders. We show in Fig. 5 three particular cases for $w=p / 2, w=p / 10$, and $w=0$. For $w=p / 10$, diffraction orders higher than firsts disappears and for $w=p / 2$ only zeroth order remains.

\section{NUMERICAL SIMULATIONS}

To corroborate the analytical results, we perform in this section several numerical simulations using a fast-Fourier-transform based direct integration method that uses the Rayleigh-Sommerfeld approach, 23. Firstly, we define the random diffraction grating as an ensemble of slits placed almost periodically along the $x$-axis. We introduce the randomness into the position of every slit as a random displacement around the nominal position (periodical), following a gaussian distribution. To understand the propagation process, we show in Fig. 6 four examples of propagation with different amounts of randomness, (a) $w=0,(b) w=p / 10, \quad(c) w=p / 5$, and $(d) w=p / 2$. It shows how the self-imaging process is harmed by the randomness of the grating. Anyway, to compare with the analytical results, we need to perform an average over an ensemble of simulations. Thus, we show in Fig. 7 the intensity at the near field produced by an ensemble of 1000 gratings with the same statistical parameters. Self-images are smoother for low randomness level. On the contrary, $w=p / 2$ corresponds with high randomness limit and produces the total disappearance of the selfimages at the near field, Fig. 7c. We calculate the contrast by using the definition given in Eq. (12), Fig. 4 (dash line). Numerical results are comparable to analytical results and corroborate the obtained analytical formalism.
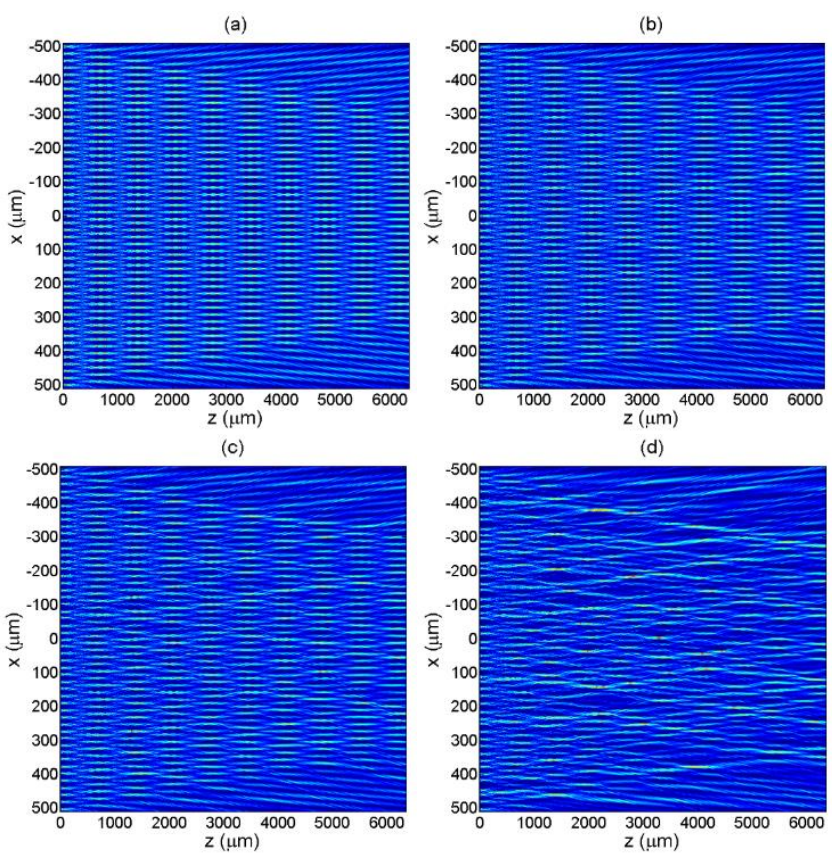

Fig. 6. Examples of diffracted intensity (one realization) illuminated by a plane wave of $\lambda=630 \mathrm{~nm}$, (a) $w=0$, (b) $w=p / 10$, (c) $w=p / 5$, and (d) $w=p / 2$. The nominal period of the grating is $p=20 \mu \mathrm{m}$.
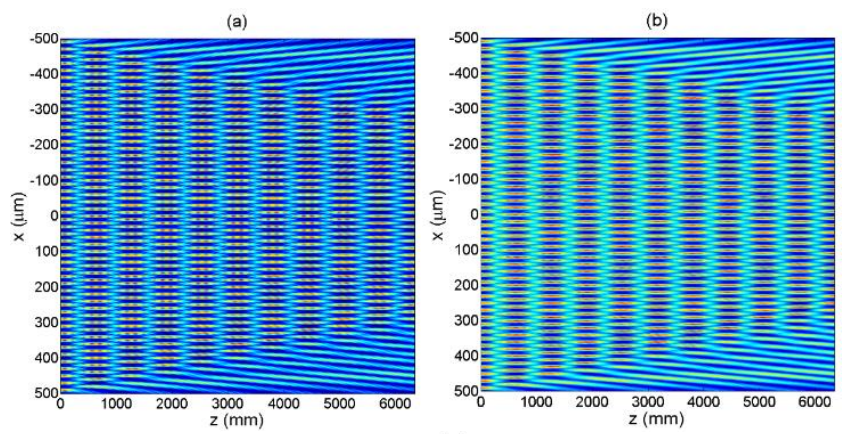

(c)

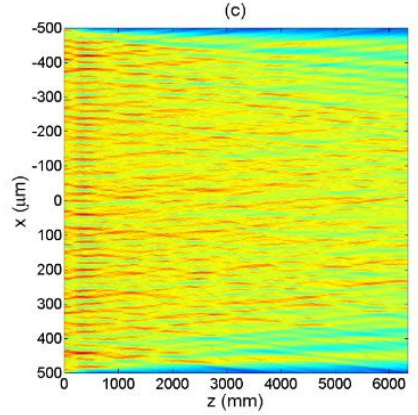

Fig. 7. Examples of average intensity produced by an ensemble of 1000 gratings with the same statistical parameters and illuminated by a plane wave of $\lambda=630 \mathrm{~nm}$, (a) $w=p / 10$, (b) $w=p / 5$, and (c) $w=p / 2$. The nominal period of the grating is $p=20 \mu \mathrm{m}$.

\section{CONCLUSIONS}

In this work, we analyze the diffraction produced by random Ronchi diffraction gratings where randomness is present in the position of the slits. This manufacture error could be common in fabrication processes and, up to our knowledge, it has not been analyzed yet. We give analytical results of the behaviour of this kind of gratings in the near and 
far field and corroborate them by numerical analysis. Randomness produces a smoothing of the self-images and elimination of harmonics depending on the amount of randomness. These results are useful in applications in which manufacture errors have to be taken into account.

Funding Inforamtion. Project art. 83 num. 52-2016 UCM/Fagor Automation S. Coop.

Acknowledgment. In memoriam of J. M. Rico-Garcia. The authors thank A. Luis for his inspirational and encourage comments.

\section{References}

1. C. Palmer and E. Loewen Diffraction grating handbook 2005 (New York: Richardson Grating Loboratory)

2. A. W. Lohmann and D. E. Silva, "An interferometer based on the Talbot effect," Opt. Commun. 2, 413-415 (1971).

3. G. Schirripa-Spagnolo, G. Ambrosini, and D. Paoletti, "Displacement measurement using the Talbot effect with a Ronchi grating," J. Opt. A: Pure Appl. Opt. 4, S376-S380 (2002).

4. B. F. Oreb and R. G. Dorsch, "Profilometry by phase-shifted Talbot images," Appl. Opt. 33, 7955-7962 (1994).

5. S. Wei, S. Wu, I. Kao, and F. P. Chiang, "Measurement of wafer surface using shadow moire technique with Talbot effect," Trans. ASME J. Electron. Packag. 120, 166-170 (1998).

6. L. M. Sanchez-Brea, F. J. Torcal-Milla, and E. Bernabeu, "Far field of gratings with rough strips," J Opt. Soc. Am. A 25, 828-833 (2008).

7. F. J. Torcal-Milla, L. M. Sanchez-Brea, and E. Bernabeu, "Self-imaging of gratings with rough strips," J Opt. Soc. Am. A 25, 2390-2394 (2008).

8. F. J. Torcal-Milla, L. M. Sanchez-Brea, and E. Bernabeu, "Double grating systems with one steel tape grating," Opt. Commun. 281, 5647-5652 (2008).

9. F. J. Salgado-Remacha, F. J. Torcal-Milla, L. M. Sanchez-Brea, and E. Bernabeu, "Use of steel substrates in diffractive optics: Near field of high surface quality steel tape gratings," Opt. Laser Eng. 49, 356-360 (2011).

10. M. J. Madou "Manufacturing techniques for microfabrication and nanotechnology 2011 (Boca Raton: CRC Press,)

11. F. J. Torcal-Milla, L. M. Sanchez-Brea, and E. Bernabeu "Diffraction of gratings with rough edges," Opt. Express 16, 19757-19769 (2008).

12. J. M. Rico-Garcia and L. M. Sanchez-Brea "Binary gratings with random heights," Appl. Opt. 48, 3062-3069 (2009).

13. F. J. Torcal-Milla, L. M. Sanchez-Brea, and E. Bernabeu "Talbot effect with rough reflection gratings," Appl. Opt. 46, 3668-3673 (2007).

14. L. M. Sanchez-Brea, F. J. Torcal-Milla, and E. Bernabeu "Talbot effect in metallic gratings under Gaussian illumination," Opt. Commun. 278, 23-27 (2007).

15. L. M. Sanchez-Brea and F. J. Torcal-Milla "Near-field diffraction of gratings with surface defects," App. Opt. 49, 2190-2197 (2010).

16. Y. Lu, C. Zhou, and H. Luo "Talbot effect of a grating with different kind of flaws," J Opt. Soc. Am. A 22, 2662-2667 (2005).

17. A. Lin and J. Phillips "Optimization of random diffraction gratings in thinfilm-solar cell using genetic algorithms," Sol. Energy Mat. Sol. C 92, 16891696 (2008).

18. W. H. F. Talbot "Facts relating to optical science," Philos. Mag. 9, 401-407 (1836).

19. K. Patorski "The self-imaging phenomenon and its applications," Prog. Opt. 27, 1-108 (1989).

20. E. Keren and O. Kafri "Diffraction effects in moire deflectometry," J. Opt. Soc. Am. A 2, 111-120 (1985).

21. Goodman J W 2000 Statistical Optics (New York: John Wiley and Sons Inc.)

22. F. J. Torcal-Milla, L. M. Sanchez-Brea, and J. Vargas "Effect of aberrations on the self-imaging phenomenon," J. Lightwave Technol. 29, 1051-1057 (2011).
23. F. Shen and A. Wang "Fast-Fourier-transform based numerical integration method for the Rayleigh-Sommerfeld diffraction formula," Appl. Opt. 45, $1102-1110$ (2006). 